TITLE:

\title{
Experimental Evidence of a Twofold Electromagnetic Enhancement Mechanism of Surface-Enhanced Raman Scattering
}

\section{$\operatorname{AUTHOR}(S)$ :}

Kumar, Samir; Tokunaga, Kouta; Namura, Kyoko; Fukuoka, Takao; Suzuki, Motofumi

\section{CITATION:}

Kumar, Samir ... [et al]. Experimental Evidence of a Twofold Electromagnetic

Enhancement Mechanism of Surface-Enhanced Raman Scattering. Journal of Physical Chemistry C 2020, 124:21215-21222

\section{ISSUE DATE:}

2020-09-24

URL:

http://hdl.handle.net/2433/258965

\section{RIGHT:}

This document is the Accepted Manuscript version of a Published Work that appeared in final form in Journal of Physical Chemistry C, copyright $\odot$ American Chemical Society after peer review and technical editing by the publisher. To access the final edited and published work see https://doi.org/10.1021/acs.jpcc.0c07930.; The full-text file will be made open to the public on 1 September 2021 in accordance with publisher's 'Terms and Conditions for SelfArchiving': この論文は出版社版でありません。引用の際には出版社版をご確認ご利用ください。; This is not the published version. Please cite only the published version. 


\title{
Experimental Evidence of Twofold Electromagnetic
}

\section{Enhancement Mechanism of Surface-Enhanced}

\section{Raman Scattering}

Samir Kumar*, Kouta Tokunaga, Kyoko Namura, Takao Fukuoka, and Motofumi Suzuki*

Department of Micro Engineering, Graduate School of Engineering, Kyoto University, Katsura, Nishikyo, Kyoto 615-8540 Japan.

KEYWORDS: Metals; Raman spectroscopy; electromagnetic enhancement; plasmons

\begin{abstract}
The electromagnetic enhancement mechanism is a major contributor to surfaceenhanced Raman-scattering enhancements, which are a direct consequence of the roughness present on noble metal surfaces. The electromagnetic enhancement mechanism is a two-fold phenomenon that involves the enhancement of both the incident excitation and scattered Raman fields. In this paper, we report a direct observation of the double-enhancement mechanism using an $\mathrm{Ag}$ nanorod array/ $\mathrm{SiO}_{2}$ dielectric layer/Ag mirror multilayer thin-film "local plasmon resonator." The effect of light interference was controlled by adjusting the film thickness of the $\mathrm{SiO}_{2}$ phase control layer ( $\mathrm{PCL}$ ), and the absorption rate in the Raman-scattering wavelength range was tuned from $90 \%$ to $0 \%$. In addition to the characteristic Raman peak of an aqueous solution of 4,4-bipyridine, the background of the Raman-scattering spectrum was also enhanced. By examining the relationship between the background Raman emission and the absorption spectrum,
\end{abstract}


we demonstrated that the intensity of the background emission is closely related to the surfaceenhanced Raman scattering (SERS) enhancement. We further illustrated that the Raman-scattered field and background were enhanced when the absorption was high in the wavelength range of the scattering field. The present results not only suggest that the PCL layer may increase the intensity of plasmon-mediated broadband emission but also provide us with sufficient evidence for a twofold SERS enhancement mechanism.

\section{INTRODUCTION}

Surface-enhanced Raman scattering (SERS) refers to an enormous enhancement in the Raman scattering intensity from molecules on the surface of noble metal particles and can be $10^{8}$ times or more than that of free molecules. ${ }^{1-3}$ Numerous enhancement mechanisms were proposed during the initial days of SERS. ${ }^{4,5}$ However, only two mechanisms are currently widely accepted, namely, the chemical enhancement (CE) theory and the electromagnetic (EM) theory. ${ }^{6,7}$ The CE theory is based on the chemical interactions between the adsorbed molecules and the noble metal and has a maximum contribution of approximately two to three orders of magnitude. ${ }^{8,9}$ The EM theory, on the other hand, considers the molecule as a point dipole $\mu$ that responds to the enhanced local fields at or near the metal surface; these fields couple the incident field $E_{0}$ to the surface plasmons. ${ }^{10}$ The presence of nanostructured metal surfaces can alter the Raman-scattering process in two ways. ${ }^{11-}$ 14 The first step involves the enhancement of the excitation field at the nanoparticle surface. Localized surface plasmon resonance (LSPR) generates a local electric field near the metal nanoparticles. The molecules around the metal nanoparticles are subjected to an enhanced electric field, thereby enhancing Raman scattering. This increase in the local electric field at the metallic surface is termed local field enhancement. The second step involves the enhancement of the Raman-scattered light from the adsorbed molecule. This enhanced Raman scattering can also 
partially excite the LSPR of metal nanoparticles, further enhancing the Raman-scattered light, and is known as radiation enhancement. The SERS enhancement factor (EF) is generally expressed as a product of these two electromagnetic enhancement mechanisms: ${ }^{7}$

$$
E F=\frac{\left|E_{\text {out }}\right|^{2}\left|E_{\text {out }}^{\prime}\right|^{2}}{\left[\left.E_{0}\right|^{4}\right.}
$$

where $E_{0}^{2}$ is the incident field intensity, $E_{\text {out }}^{2}$ is the magnitude of the electromagnetic field outside the particle, and $E_{\text {out }}^{\prime 2}$ is the scattered field intensity. If the Stokes shift is small, both $E_{0}$ and $E_{0}^{\prime}$ are at approximately the same wavelength. This two-fold enhancement (the enhancement of both the incident and Raman fields) is described in the literature and is commonly referred to as $E^{4}$ enhancement, as illustrated in Figure 1(a).

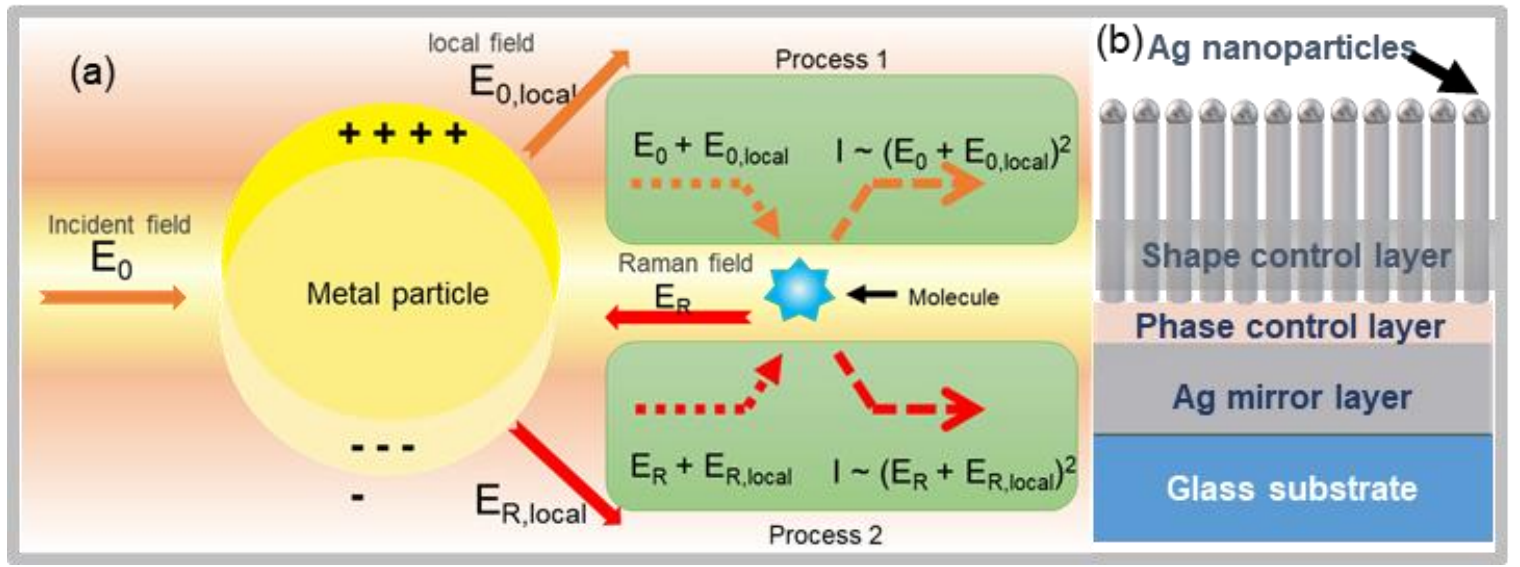

Figure 1. Schematic of (a) twofold electromagnetic SERS enhancement and (b) multilayer thin-film "local plasmon resonator."

A few reports in the literature support the SERS twofold EM process. ${ }^{15-18}$ Yoshida et al. examined the surface-enhanced resonance Raman-scattering (SERRS) EM theory by calculating the total emission spectra and varying the EM enhancement factors, Raman and fluorescence spectra, and excitation wavelengths. ${ }^{19}$ They observed that the experimental total emission spectra were consistent with the calculated total emission spectra as a function of these parameters. Nevertheless, their analysis was inconclusive because they assumed that the accuracy of their 
experimental and calculated transmission spectra would offer strong evidence that the SERRS-EM theory was quantitatively accurate. McFarland et al. reported the study of benzenethiol adsorbed by Ag nanoparticle arrays fabricated by nanosphere lithography (NSL) using a technique referred to as wavelength-scanned surface-enhanced Raman excitation spectroscopy. ${ }^{18}$ They studied three vibrational modes of benzenethiol $\left(1575,1081\right.$, and $\left.1009 \mathrm{~cm}^{-1}\right)$ and concluded that the smaller Raman-shifted peak reflected a maximum enhancement closer to the LSPR than that of the larger Raman-shifted peak. That is, the highest SERS enhancement occurred at slightly shorter excitation wavelengths than that of LSPR, such that both incident and Raman-scattered photons were strongly enhanced. However, their objective was to explore the optimal wavelength of excitation as a function of the spectral position of the maximum LSPR extinction, and their method required expensive and sophisticated instrumentation, in which both the SERS excitation and detection system could be tuned. Khlebtsov et al. studied SERS from Au nanorods (AuNRs) as a function of their aspect ratio and shape. ${ }^{20}$ However, their observation contradicts the current understanding of the electromagnetic contribution, namely that the highest SERS response is obtained with the excitation laser wavelength at approximately the plasmon resonance (PR). In contrast, they obtained comparable intensities of characteristic peaks for all samples, regardless of the plasmonic peak positions of the AuNRs. Although the contribution of the EM mechanism to the overall SERS enhancement is well established, to the best of our knowledge, direct experimental evidence for a twofold EM enhancement mechanism of SERS has not yet been reported.

To verify the two-fold enhancement mechanism of SERS, it is necessary to prepare metal nanoparticles that exhibit different responses to excitation and scattered light (Equation 1). This can be realized using a wavelength-tunable laser for the excitation light, but because the diffraction efficiency of the spectroscope and the sensitivity of the photodetector depend on the wavelength, 
quantitative verification is not an easy task. Several methods have been proposed to produce ordered noble metal nanostructures with well-defined morphologies and optical responses. ${ }^{21-23}$ Previously, we have reported Ag nanorod arrays (AgNRAs) on a $\mathrm{SiO}_{2}$ shape control layer (SCL) with a strong anisotropic surface morphology using the dynamic oblique angle deposition (OAD) method. ${ }^{24-26}$ By varying the thickness of the SCL, the aspect ratio of the Ag nanorods can be changed; therefore, the PR can be tuned to the wavelength region between 750 and $950 \mathrm{~nm} .^{27,28}$ Consequently, the response to the excitation light and the Raman-scattered light can be changed to a certain extent. However, varying the thickness of the SCL can also change the size, shape, and arrangement of the nanostructures, which can significantly alter the SERS and is not suitable for verifying the two-fold enhancement mechanism. That is validation of this mechanism would require metal nanostructures with the same shape and size but different responses to excitation and scattered light. This motivated our study, in which we fabricated an $\mathrm{AgNRAs} / \mathrm{SiO}_{2}$ dielectric layer/Ag mirror multilayer thin-film "local plasmon resonator" (MLPR), as illustrated in Figure 1. By varying the thickness of the flat and dense phase control layer (PCL), the optical path length of the light in the multilayer film can be precisely controlled, and the light can be confined by the AgNRAs owing to interference.

This work enabled us to confirm the two-fold EM enhancement mechanism of SERS by measuring the SERS characteristics of MLPR at different wavelengths and analyzing the optical absorption and Raman-scattering spectra for excitation and scattered light. A close relationship between the absorption and SERS enhancement near the Raman-scattered wavelengths is a direct indication of the role of scattered light in the generation of SERS.

\section{MATERIALS AND METHODS}

\subsection{Fabrication of the Multilayer Local Plasmon Resonator}


The MLPR was fabricated using a dynamic OAD technique, in which the deposition angle and/or in-plane direction of the substrate can be changed during deposition, thus allowing the threedimensional morphology to be controlled. ${ }^{29-31}$ The detailed fabrication process can be found elsewhere. ${ }^{32,33}$ The PCL thickness was adjusted in the range of 0-210 nm. Subsequently, the $\mathrm{SiO}_{2}$ SCL with anisotropic surface morphology was deposited using the serial bideposition (SBD) method. ${ }^{33,34}$ The vapor deposition angle $\alpha$ for $\mathrm{SiO}_{2}$ was fixed at $78.6^{\circ}$, and the in-plane angle was swiftly rotated by $180^{\circ}$ after each fixed amount of vapor deposition. SCL of thickness $d_{\mathrm{SCL}}=576$ $\mathrm{nm}$ was prepared using 30 cycles of SBD. Subsequently, Ag was deposited up to a thickness $t_{A g}$ of $8 \mathrm{~nm}$ at a deposition angle, $\alpha_{A g}$, of $73.4^{\circ}$ onto the fabricated SCL layer. The in-plane angle was not changed during the deposition of Ag. The amount of Ag that was deposited was sufficiently low to maintain the discontinuous island structure of $\mathrm{Ag}$ on the SCL.

\subsection{Optical and SERS Characterization}

The reflectance with polarized light for the MLPR was measured in both air and water. For the measurement of reflectance, the angle of incidence was suitably small to be considered normal incidence. A deuterium light source (DH-2000, Ocean Optics) was used in the ultraviolet light region, and a halogen light source (LS-1, Ocean Optics) was used for reflectance measurements in the visible light region. The light guided by the optical fiber was collimated by a convex lens and was incident on the MLPR sample perpendicular to the sample plane. The reflectance spectra were collected using a spectrometer (Ocean Optics USB4000). Reflectance measurements were performed for two mutually orthogonal polarizations termed "s" (light polarized parallel to the long axis of the Ag nanoparticles) and " $p$ " (light polarized perpendicular to the long axis of the Ag nanoparticle axis). The reflectance in water was measured by placing a 0.5 -mm-thick silicone cell with a $5 \times 5 \mathrm{~mm}$ opening in the center on the sample, $15 \mu \mathrm{L}$ of pure water was injected into the 
silicone cell opening, and the sample was sealed with a cover glass. The method that was used to measure the reflection in water was similar to that used to measure it in air, unless specified otherwise.

The in situ SERS measurement was carried out by constructing a small cell (with a diameter of $3.6 \mathrm{~mm}$ and containing a spacer of silicone rubber with a thickness of $0.5 \mathrm{~mm}$ ). The MLPR was placed inside the cell and, after filling it with a $10 \mu \mathrm{L}$ droplet of an aqueous solution of $1 \mathrm{mM} 4,4$ bipyridine (BPY), sealed with a cover glass. The SERS measurements were conducted using an inverted Raman spectrometer (RAM200S; LambdaVision Inc.) with a 10× objective lens. A 785 $\mathrm{nm}$ laser with an intensity of $30 \mathrm{~mW}$ was utilized as the light source for excitation. In addition, SERS measurements were performed using an s-polarized laser with a polarizing film placed near the objective lens.

\section{RESULTS AND DISCUSSION}

\subsection{Morphology of the Multilayer Local Plasmon Resonator}

Figure 2 presents the top and cross-sectional SEM micrographs for the MLPR with $d_{\mathrm{PCL}} \approx 120$ nm. The white and orange arrows represent the directions of the $\mathrm{Ag}$ and $\mathrm{SiO}_{2}$ vapor during deposition, respectively. The MLPR has AgNRAs/ $\mathrm{SiO}_{2} \mathrm{SCL} / \mathrm{SiO}_{2} \mathrm{PCL} / \mathrm{Ag}$ mirror surface layers. When light falls onto a multilayer thin-film structure with several different materials, multiple reflections occur within the structure. The multiple reflections can be coherent, and they interact with each other depending on the light source and thickness of the layer. It is crucial to control the distance between the AgNRAs and the mirror surface to precisely adjust the light coupled to the local plasmons. Earlier, we illustrated that the reflectance from the nanostructures under consideration changes significantly owing to the interference. ${ }^{35}$ When light is incident on the MLPR, it is reflected from the Ag mirror surface, and the reflected light is reflected again by the 
AgNRAs after passing through the dielectric layer. These two reflections can interfere constructively (accumulating the energy of the incident light onto the Ag nanoparticles) or destructively (in the absence of interaction between the incident light and Ag nanoparticles) depending on the thickness of the PCL $d_{\mathrm{PCL}}$.

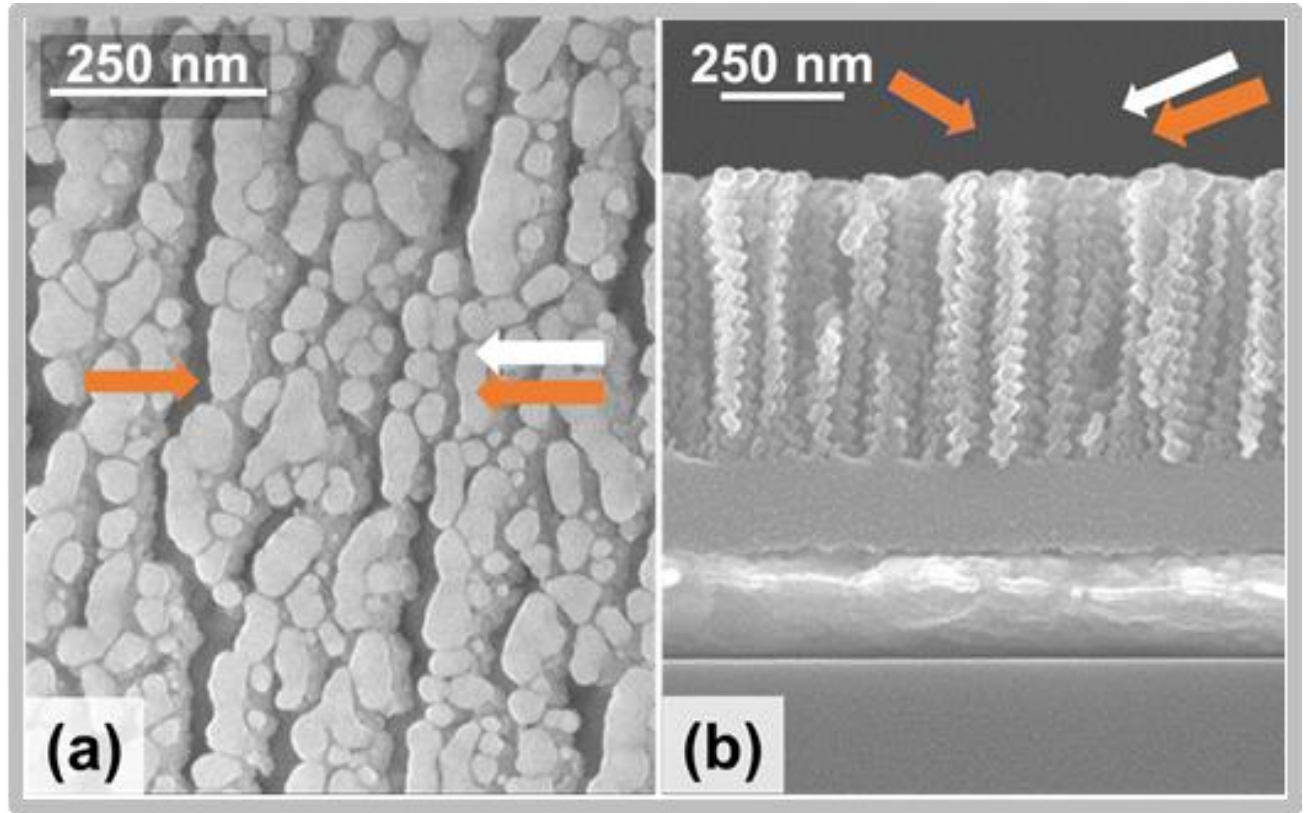

Figure 2. (a) Top view and (b) cross-sectional SEM image of MLPR sample with PCL thickness of $120 \mathrm{~nm}$. The orange arrows represent the direction of $\mathrm{SiO}_{2}$ vapor deposition, whereas the white arrows represent the direction of Ag deposition.

Similarly, in the case of scattered light, the interaction with Ag nanoparticles can be tuned by the effect of interference. Hence, this dielectric layer is referred to as a PCL, as it controls the optical path length of light. A zigzag dielectric $\mathrm{SiO}_{2}$ layer with an anisotropic surface morphology was deposited on the PCL layer, and Ag was obliquely vapor-deposited thereon, such that the Ag vapor was deposited only on the irregular portion of the template. The plasmonic properties of the AgNRAs depend strongly on the thickness of the underlying $\mathrm{SiO}_{2} \mathrm{SCL}$ because the shape of the nanorods reflects the anisotropic surface morphology of the SCL. ${ }^{25}$ Therefore, elongated AgNRs 
reflecting the anisotropic surface morphology of the $\mathrm{SiO}_{2}$ template are formed, as depicted in Figure 2(a). An $\mathrm{SiO}_{2}$ template that controls the shape, such as the aspect ratio and size of the $\mathrm{Ag}$ nanoparticles, is termed an SCL.

The light phase can be controlled by changing the thickness of the flat and uniform PCL, whereas the surface morphology of the Ag nanoparticles can remain unaffected by maintaining the SCL thickness constant. This is an essential feature of the local plasmon resonator. The cross-sectional SEM image confirms the laminated structure of the AgNRAs/SCL/PCL/Ag mirror MLPR. The SCL consists of a zigzag $\mathrm{SiO}_{2}$ nanostructure and discontinuous prolate-shaped AgNRAs, with their long axis in the direction perpendicular to the vapor deposition flow. In earlier studies, we examined the growth of the AgNRAs in greater detail. ${ }^{29,36}$ The AgNRs and SCL have unique morphologies and are physically self-organized owing to self-shadowing during oblique vacuum deposition. The fan-out effect arising from the anisotropy of the shadowing effect causes anisotropic broadening of the $\mathrm{SiO}_{2}$ nanocolumns. ${ }^{37}$ The columns form a highly elliptical crosssection perpendicular to the plane of the incident vapor flux. Thus, the AgNRAs grow on top of the $\mathrm{SiO}_{2}$ columns elongated in the direction perpendicular to the $\mathrm{Ag}$ deposition plane with a quasiparallel alignment.

\subsection{Optical Properties of the Local Plasmon Resonator}

Figure 3(a) shows the polarization-dependent reflectance spectra as a function of $d_{\mathrm{PCL}}$ in air. Multiple reflections within the films attributed to interference processes are characterized by intermittent dark and light patterns. The brighter parts indicate reflection lower than those represented by the darker parts. As can be observed, the reflectance spectra exhibit a strong polarization dependence owing to the quasi-prolate morphology of the AgNRAs. Clearly, for a fixed value of $d_{P C L}$, the reflectance changed periodically as a function of the photon energy, and 
the reflectance in the NIR region for $s$-polarized light was much higher than that for the $p$-polarized light. Focusing on the reflectance in the energy range (1.3-1.5 eV) of Raman scattering and the excitation laser $(\lambda=785 \mathrm{~nm})$, anti-reflective conditions for the s-polarized light were found where the reflectance was negligibly small. In the range of $100 \mathrm{~nm} \leq d_{P C L} \leq 140 \mathrm{~nm}$, the absorbance changed from approximately $90 \%$ to $0 \%$ for the $s$-polarized light. However, for the $p$-polarized light, the absorbance was almost constant $(\sim 60 \%)$ within the same wavelength range.

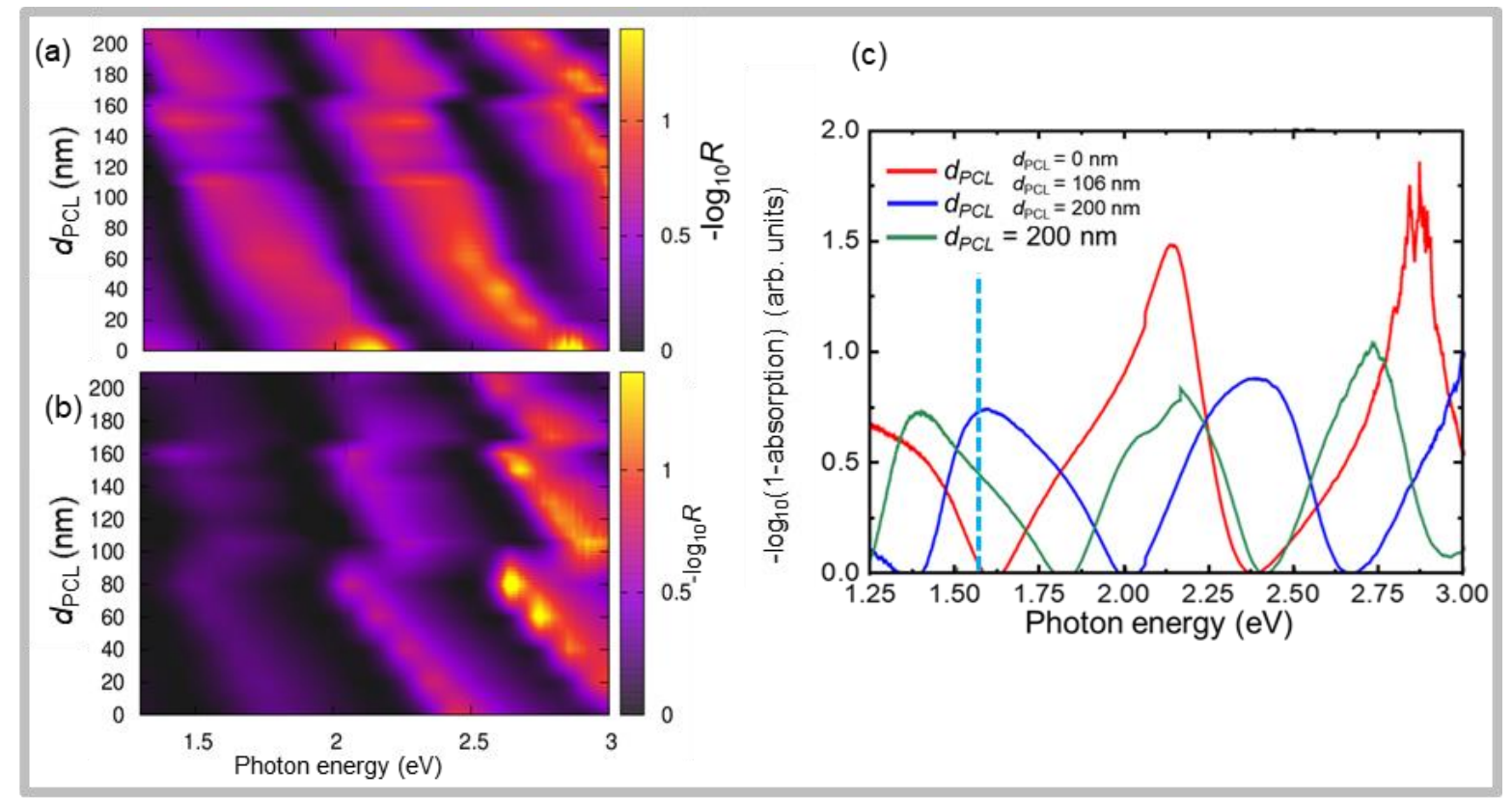

Figure 3. (a) $s$-polarized and (b) p-polarized reflectance for $d_{\mathrm{PCL}}$ in the range $0-210 \mathrm{~nm}$; (c) comparison of s-polarized reflectance spectra for $d_{\mathrm{PCL}}=0,106$, and $200 \mathrm{~nm}$.

Figure 3(c) presents the variations in absorbance for $d_{P C L}=0,106$, and $200 \mathrm{~nm}$ for $s$-polarized light. Near the excitation photon energy $(1.57 \mathrm{eV}$, blue dotted line), most of the light is reflected for $d_{\mathrm{PCL}}=0 \mathrm{~nm}$, but for $d_{\mathrm{PCL}}=106 \mathrm{~nm}$, there is an absorption peak of approximately $85 \%$, and for $d_{\mathrm{PCL}}=200 \mathrm{~nm}$, the absorption has a value of approximately $55 \%$. In other words, a change in the PCL thickness leads to a change in the optical path length, thereby leading to different conditions 
for interference. Thus, we can tune the absorbance by changing the PCL thickness, and this forms the most important characteristic of the local plasmon resonator.

Figure 4 presents a comparison of the absorption in air and water with $s$-polarized light for $d_{\mathrm{PCL}}=$ $20 \mathrm{~nm}, 110 \mathrm{~nm}$, and $200 \mathrm{~nm}$. The absorption spectrum in water is red-shifted by approximately 50 $\mathrm{nm}$ in the short-wavelength range and by approximately $100 \mathrm{~nm}$ in the long-wavelength range as compared to that in air. This shift in the absorption in water can be explained by considering the difference in the refractive indices of air and water (the optical contrast), which has a strong effect on interference. It is well established that for a multilayer system, the phase shift at transmission increases monotonically as a function of the wavelength. ${ }^{38}$ Another reason may be that when this multilayer film is immersed in a fluid, the fluid can enter the voids in the SCL nanostructures and the effective refractive index of the SCL medium may change; this may result in a change in the effective optical thickness of the layer. ${ }^{39}$ A variation in the optical thickness of the non-absorbing multilayer may result in the displacement of the reflection spectra.
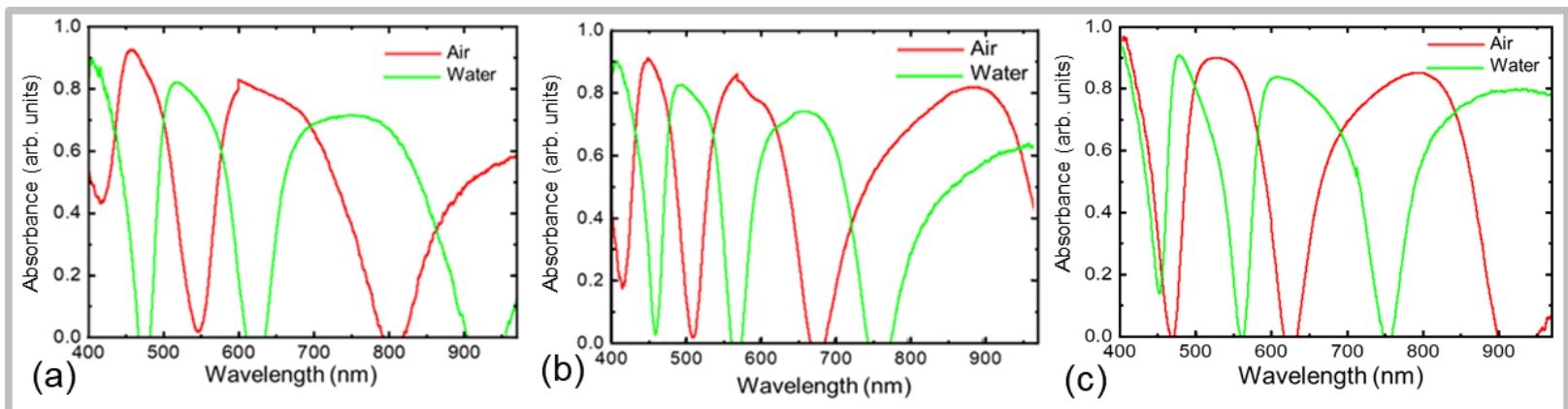

Figure 4. s-polarized absorption spectra in air and water for $d_{\mathrm{PCL}}=$ (a) 20, (b) 110, and (c) 200 nm.

\subsection{SERS Study of the Multilayer Local Plasmon Resonator}

Figure 5 shows the SERS color map for different values of $d_{\mathrm{PCL}}$. The vertical axis represents the values of $d_{P C L}$, and the different colors represent the intensity of the SERS spectrum of BPY with the s-polarized $785 \mathrm{~nm}$ laser. Red and dark black colors indicate strong and weak 
SERS intensity, respectively. Strong SERS peaks are observed around $1000 \mathrm{~cm}^{-1}, 1290 \mathrm{~cm}^{-1}$, and $1600 \mathrm{~cm}^{-1}$; these are the characteristic SERS peaks of the BPY aqueous solution appearing at 1013 $\mathrm{cm}^{-1}, 1292 \mathrm{~cm}^{-1}$, and $1609 \mathrm{~cm}^{-1}$. The SERS intensity was minimal for $d_{\mathrm{PCL}}=0 \mathrm{~nm}$ and $200 \mathrm{~nm}$. The SERS intensity depends on the value of $d_{P C L}$ and increases when $80 \mathrm{~nm} \leq d_{P C L} \leq 150 \mathrm{~nm}$.

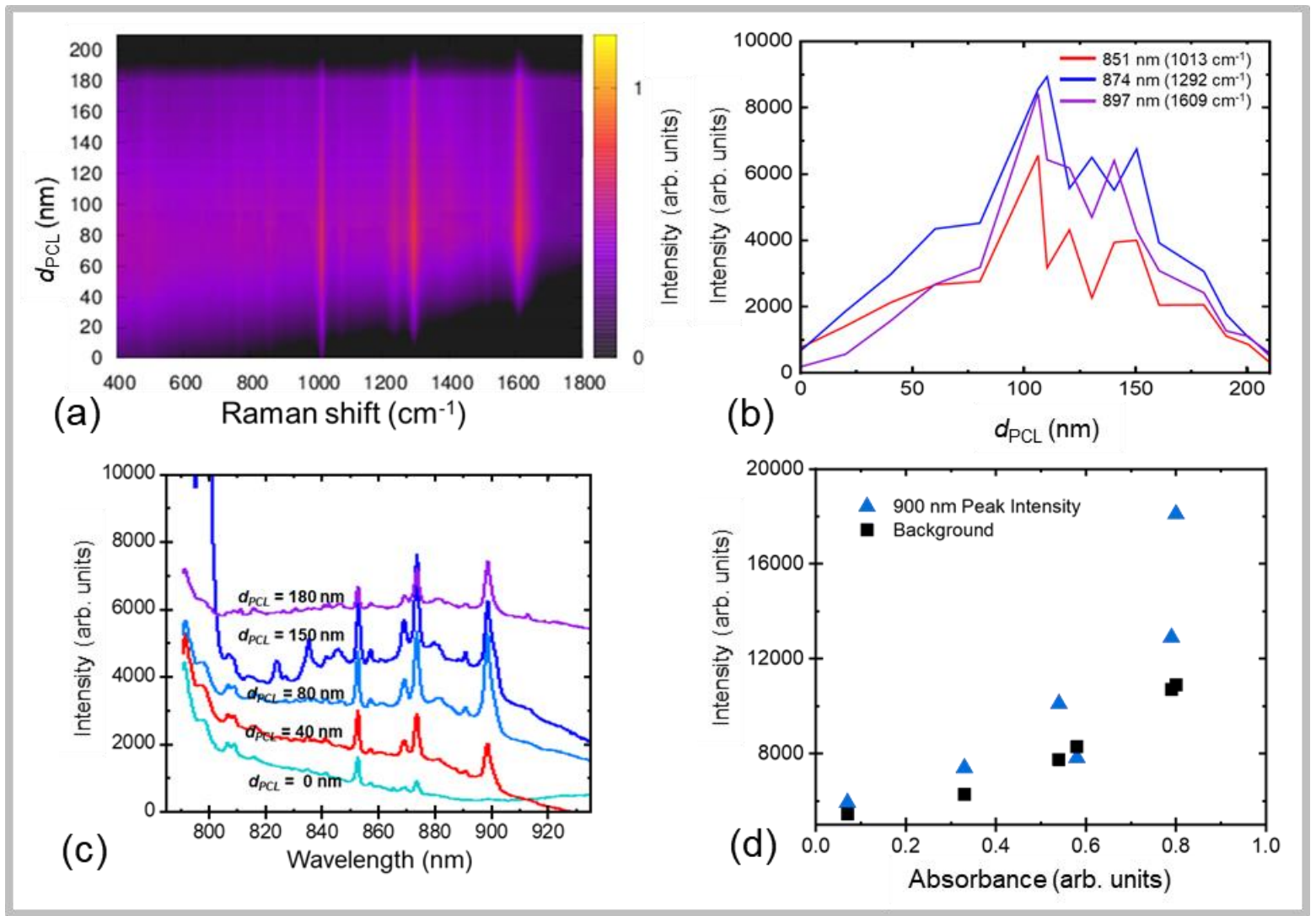

Figure 5. (a) SERS map at different values of $d_{\mathrm{PCL}}$; (b) SERS characteristic peaks intensities as a function of $d_{\mathrm{PCL}}$; (c) SERS spectra for different values of $d_{\mathrm{PCL}}$; (d) SERS peak and background intensity around $900 \mathrm{~nm}$ as a function of absorbance.

When correlated with the corresponding reflectance spectra presented in Figure 3(a), SERS was confirmed to be strong for the samples with lower reflectance. Figure 5(b) presents the SERS characteristic peaks $\left(1013,1292\right.$, and $\left.1609 \mathrm{~cm}^{-1}\right)$ as functions of $d_{\mathrm{PCL}}$. The peak intensity was maximum for $100 \mathrm{~nm} \leq d_{\mathrm{PCL}} \leq 120 \mathrm{~nm}$ and minimum for $d_{\mathrm{PCL}}=0$ and $200 \mathrm{~nm}$. The peak intensity at $d_{\mathrm{PCL}} \approx 110 \mathrm{~nm}$ was approximately 50 times stronger than that at $d_{\mathrm{PCL}}=200 \mathrm{~nm}$. In general, the 
intensity of the scattered light and absorption are good indicators of the strength of the induced dipole, as a dipole induced in metal nanoparticles by incident light either radiates the scattering light or absorbs the incident light. However, in our case, the intensity of the scattered light strongly depends on the interference conditions; thus, only the absorption corresponds to the strength of the induced dipole. Under antireflection conditions, the incident light was confined within the multilayered structures, and most of the light was absorbed by the Ag nanorods. Therefore, the enhanced local electric field close to the Ag nanorods would significantly increase the strength of the SERS.

In addition, broad continuous background enhancement was also observed in conjunction with the enhanced Raman bands. ${ }^{2,40}$ Figure 5(c) presents the SERS spectrum for various values of $d_{\text {PCL. }}$. It was observed that not only the Raman-scattering peak but also the background was enhanced, and the peak intensity depended on the film thickness, that is, on the absorption profile. The background emission intensity is closely associated with the enhancement of SERS. ${ }^{41}$ Recently, it was suggested that the continuum observed in SERS might be related to plasmon-coupled emission. Mahajan et al. demonstrated the plasmon dependence of the background in the SERS spectra and proposed electromagnetic and chemical contributions to the generation of the background. ${ }^{42}$ Farcau et al. also demonstrated that the enhanced EM fields are responsible for amplifying both the SERS and background scattering. ${ }^{43}$ Recently, Khlebtsov et al. reported the correlation between the absorption and background emission spectra. They reported that the SERS background spectra could be attributed to both the photoluminescence from the AuNR ensemble and the elastic light scattering of a very weak laser background by the same AuNR ensemble. Figure 5(d) presents the variation in the SERS peak and background at approximately $900 \mathrm{~nm}$ as a function of absorbance. At this wavelength, the SERS peaks and background were both found to 
increase with increased absorption. The background enhancement was observed to be strongly correlated with the absorbance spectra (Figure S1, Supplementary Information). In addition, the background was found to be independent of the BPY concentration (Figure S2, Supplementary Information). This confirms that the absorption corresponds to the strength of the induced dipole. Thus, the generation of the SERS background would seem to be attributable to a plasmonic mechanism in addition to the enhancement of the Raman-scattered light.

In Section 3.4, we compare the SERS spectrum with the absorbance in water to verify the enhancement of the electric field owing to Raman-scattered light.

\subsection{SERS Double Enhancement}

Figure 6 presents the absorption and SERS spectra for different values of $d_{\mathrm{PCL}}$. For $d_{\mathrm{PCL}} \leq 60 \mathrm{~nm}$ in the wavelength range of $800-900 \mathrm{~nm}$, both the absorbance and background intensities of the SERS spectrum decreased monotonically, as depicted in Figure. 6(a-c). For $d_{\mathrm{PCL}}=20$ and $40 \mathrm{~nm}$, the absorbance increased in the wavelength range of 900-1000 nm. Consequently, the SERS background was also slightly enhanced. Thus, the enhancement/degradation of the SERS background can be directly related to the absorbance at that specific wavelength.

Figure 6(d) shows the absorption and SERS spectra for $d_{\mathrm{PCL}}=110 \mathrm{~nm}$ that exhibited sample the highest SERS intensity. The absorbance near the excitation wavelength was approximately $45 \%$, which was small compared to that of the other samples. However, the absorbance for the wavelength at which the Raman scattering peak appears was approximately $80 \%$, which was the highest among all the samples. In other words, SERS enhancement can be improved by using a light source at an excitation wavelength such that the absorption is at a slightly higher wavelength than the excitation wavelength. Figure 6(d) presents the absorption and SERS spectra for $d_{\mathrm{PCL}}=$ $150 \mathrm{~nm}$. This sample had a broad absorption peak in the wavelength range of Raman scattered 
light. It is well known that the maximum SERS intensity is achieved when the absorption enhances both the incident and scattered light. ${ }^{44}$ However, we obtained the best SERS enhancement when the absorption was the maximum for the scattered wavelength $(110 \mathrm{~nm})$. The SERS intensity for $d_{\mathrm{PCL}}=150 \mathrm{~nm}$ was approximately 4 times that for $d_{\mathrm{PCL}}=200 \mathrm{~nm}$. Furthermore, for $d_{\mathrm{PCL}}=200 \mathrm{~nm}$, the absorbance near the excitation wavelength was almost zero, and the absorbance in the Raman scattered wavelength range $(850-900 \mathrm{~nm})$ was approximately $60 \%$ at the maximum, thereby resulting in a low SERS intensity and high background. Hence, for a significant SERS enhancement, both the excitation and scattered wavelengths should have high absorbance. The effect of absorbance in the excitation and scattered wavelengths on the SERS peak and background enhancement is summarized in Table 1.

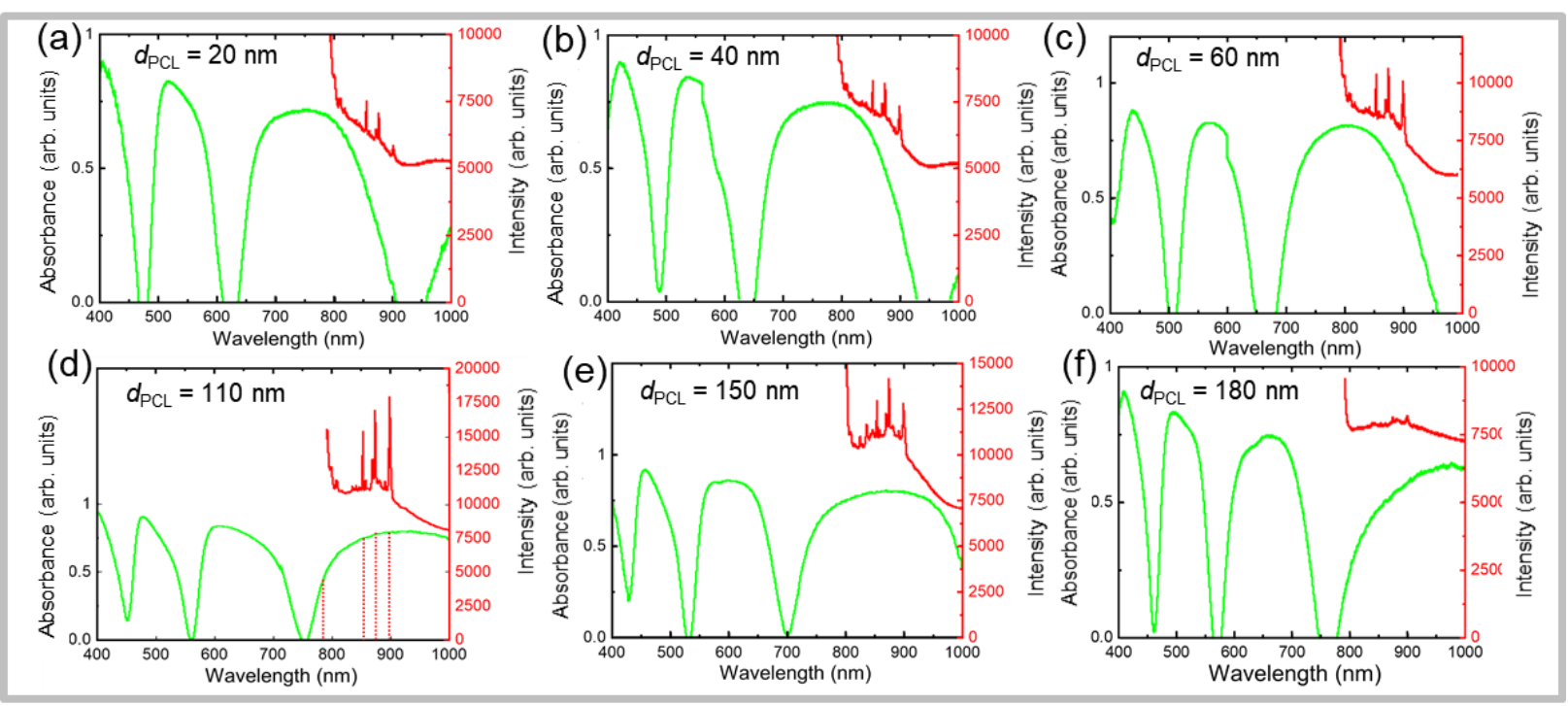

Figure 6. Absorbance and SERS spectra in water for $d_{\mathrm{PCL}}=$ (a) 20, (b) 40, (c) 60, (d) 110, (e) 150, and (f) $180 \mathrm{~nm}$. The dotted lines represent wavelengths of 785, 851, 874, and $897 \mathrm{~nm}$.

Table 1. Effect of absorbance at the excitation and scattering wavelengths on the enhancement of the SERS peak and the background 


\begin{tabular}{|c|c|c|c|c|}
\hline \multirow[b]{2}{*}{$d_{\mathrm{PCL}}(\mathrm{nm})$} & \multicolumn{2}{|c|}{$\begin{array}{c}\text { Absorbance in the wavelength } \\
\text { range }\end{array}$} & \multicolumn{2}{|c|}{ SERS } \\
\hline & $\begin{array}{c}\text { Excitation } \\
\text { range: } \\
750-800 \mathrm{~nm}\end{array}$ & $\begin{array}{l}\text { Scattering } \\
\text { range: } \\
850-900 \text { nm }\end{array}$ & $\begin{array}{c}\text { Peak } \\
\text { enhancement }\end{array}$ & $\begin{array}{l}\text { Background } \\
\text { enhancement }\end{array}$ \\
\hline 20,40 & high & low & fair & fair \\
\hline 180 & zero & medium & poor & good \\
\hline 60 & high & medium & good & good \\
\hline 150 & medium & medium & good & good \\
\hline 110 & low & high & excellent & excellent \\
\hline
\end{tabular}

*Low implies $\leq 25 \%$, medium corresponds to $\leq 50 \%$, and high infers $\leq 75 \%$ absorbance.

The SERS background was found to be correlated with the absorbance in the Raman-scattering wavelength range. The measured intensity and the spectral profile of the SERS background strongly depend on the absorbance wavelength and follow the absorbance spectral profile. Both the SERS peaks and background were most strongly enhanced when the absorbance of the scattering light was high (80\%), even for slightly low absorbance in the vicinity of the excitation wavelength. On the contrary, the highest SERS enhancement was not observed for high or medium absorbance, only near the excitation wavelength. However, the lowest background intensity was observed when the absorbance reached a minimum for the scattering wavelength. In addition, the SERS background was strong even for no absorbance in the excitation wavelength range (Figure S3, Supplementary Information). This implies that 1) the EM mechanism is a two-step process in which the scattered field is also enhanced; 2) the enhancement of the scattered field can be a dominating factor for the enhancement of the SERS peaks as well as the background. Our results agree well with those in previous reports, which showed that the maximum enhancement was observed when the excitation wavelength was higher than that of the LSPR extinction spectrum. ${ }^{45}$ Hence, we can conclude that the electric field of both the Raman-scattered light and that of the incident light is enhanced. The understanding of SERS enhancement resulting from this study is 
expected to be useful for the development of SERS substrates that demonstrate the best SERS performance. ${ }^{46,47}$

\section{CONCLUSIONS}

In conclusion, we demonstrated that the SERS peaks, background, and absorbance spectra are correlated. Strong absorbance of both the excitation light and the Raman-scattered light significantly enhanced the SERS background and peaks. A strong SERS background was observed even if strong absorbance only occurred at approximately the scattering wavelength. The enhancement of the scattered Raman field was verified using an Ag nanorod array/dielectric layer/Ag mirror layer, of which the absorption rate in the near-infrared region was changed significantly by changing the effect of light interference without changing the morphology of the $\mathrm{Ag}$ nanoparticles. The best SERS performance was obtained for $d_{\mathrm{PCL}}=110 \mathrm{~nm}$ and was approximately 50 times that of $d_{\mathrm{PCL}}=200 \mathrm{~nm}$. We enhanced the SERS response by enhancing only the scattered Raman field, which is direct proof of the two-fold SERS enhancement mechanism. Furthermore, we proved that the measured intensity and the spectral profile of the SERS background strongly depend on the magnitude of absorption near the scattering wavelength.

\section{ASSOCIATED CONTENT}

\section{Supplementary Information}

Figure S1: Absorbance, background intensity vs. PCL thickness at three Raman peaks corresponding to the wavelengths 855,875 , and $900 \mathrm{~nm}$

Figure S2: SERS spectra of BPY at various concentrations

Figure S3: Absorbance, background intensity, and SERS peak intensity at approximately $900 \mathrm{~nm}$ and at an approximate excitation wavelength $(785 \mathrm{~nm})$ as a function of the PCL thickness. 


\section{AUTHOR INFORMATION}

\section{Corresponding Author}

*SK: drsamirkumar2017@gmail.com, MS: m-snki@me.kyoto-u.ac.jp

\section{Funding Sources}

This work was supported by JST COI under Grant Number JPMJCE.1307.

\section{ACKNOWLEDGEMENT}

We thank Dr. Kosuke Ishikawa of Kyoto University for assisting us with the SEM observations.

\section{REFERENCES}

(1) Wang, D. S.; Kerker, M. Enhanced Raman Scattering by Molecules Adsorbed at the Surface of Colloidal Spheroids. Phys. Rev. B 1981, 24 (4), 1777-1790. https://doi.org/10.1103/PhysRevB.24.1777.

(2) Fleischmann, M.; Hendra, P. J.; Hill, I. R.; Pemble, M. E. Enhanced Raman Spectra from Species Formed by the Coadsorption of Halide Ions and Water Molecules on Silver Electrodes. J. Electroanal. Chem. 1981, 117 (2), $243-255$. https://doi.org/10.1016/S0022-0728(81)80086-1.

(3) Vladimir Bochenkov, Jeremy Baumberg, Mikhail Noginov, Felix Benz, Hasan Aldewachi, Silvan Schmid, Viktor Podolskiy, Javier Aizpurua, Kaiqiang Lin, Thomas Ebbesen, Alexei A Kornyshev, James Hutchison, Katarzyna Matczyszyn, Samir Kumar, Bart de Nijs, Franci, A. B. and P. N. Applications of Plasmonics: General Discussion. Faraday Discuss. 2015, 178, 435. https://doi.org/10.1039/c5fd90025e.

(4) Yamamoto, Y. S.; Ozaki, Y.; Itoh, T. Recent Progress and Frontiers in the Electromagnetic Mechanism of SurfaceEnhanced Raman Scattering. Journal of Photochemistry and Photobiology C: Photochemistry Reviews. Elsevier B.V. December 1, 2014, pp 81-104. https://doi.org/10.1016/j.jphotochemrev.2014.10.001.

(5) Furtak, T. E.; Reyes, J. A Critical Analysis of Theoretical Models for the Giant Raman Effect from Adsorbed Molecules. Surf. Sci. 1980, 93 (2-3), 351-382. https://doi.org/10.1016/0039-6028(80)90270-8.

(6) Moskovits, M. Surface-Enhanced Spectroscopy. $\quad$ Rev. $\quad$ Mod. $\quad$ Phys. $\quad$ 1985, $\quad 57 \quad$ (3), $783-826$. https://doi.org/10.1103/RevModPhys.57.783.

(7) Stiles, P. L.; Dieringer, J. A.; Shah, N. C.; Van Duyne, R. P. Surface-Enhanced Raman Spectroscopy. Annu. Rev. Anal. Chem. 2008, 1 (1), 601-626. https://doi.org/10.1146/annurev.anchem.1.031207.112814.

(8) Su, J.-P.; Lee, Y.-T.; Lu, S.-Y.; Lin, J. S. Chemical Mechanism of Surface-Enhanced Raman Scattering Spectrum of Pyridine Adsorbed on Ag Cluster: Ab Initio Molecular Dynamics Approach. J. Comput. Chem. 2013, 34 (32), $2806-2815$. https://doi.org/10.1002/jcc.23464.

(9) Lombardi, J. R.; Birke, R. L.; Lu, T.; Xu, J. Charge-Transfer Theory of Surface Enhanced Raman Spectroscopy. J. Chem. Phys. 1986, 84 (8), 4174-4180. https://doi.org/10.1063/1.450037.

(10) Gersten, J.; Nitzan, A. Electromagnetic Theory of Enhanced Raman Scattering by Molecules Adsorbed on Rough Surfaces. J. Chem. Phys. 1980, 73 (7), 3023. https://doi.org/10.1063/1.440560. 
(11) Deng, C. Y.; Zhang, G. L.; Zou, B.; Shi, H. L.; Liang, Y. J.; Li, Y. C.; Fu, J. X.; Wang, W. Z. Local Electric Field Enhancement of Neighboring Ag Nanoparticles in Surface Enhanced Raman Scattering. Adv. Mater. Res. 2013, 760-762, 801-805. https://doi.org/10.4028/www.scientific.net/AMR.760-762.801.

(12) Cialla, D.; Petschulat, J.; Hübner, U.; Schneidewind, H.; Zeisberger, M.; Mattheis, R.; Pertsch, T.; Schmitt, M.; Möller, R.; Popp, J. Investigation on the Second Part of the Electromagnetic SERS Enhancement and Resulting Fabrication Strategies of Anisotropic Plasmonic Arrays. ChemPhysChem 2010, $11 \quad$ (9), $1918-1924$. https://doi.org/10.1002/cphc.200901009.

(13) Gersten, J. I.; Nitzan, A. Electromagnetic Theory: A Spheroidal Model. In Surface Enhanced Raman Scattering; Springer US: Boston, MA, 1982; pp 89-107. https://doi.org/10.1007/978-1-4615-9257-0_5.

(14) Zeman, E. J.; Schatz, G. C. An Accurate Electromagnetic Theory Study of Surface Enhancement Factors for Silver, Gold, Copper, Lithium, Sodium, Aluminum, Gallium, Indium, Zinc, and Cadmium. J. Phys. Chem. 1987, 91 (3), $634-643$. https://doi.org/10.1021/j100287a028.

(15) Xu, H.; Aizpurua, J.; Käll, M.; Apell, P. Electromagnetic Contributions to Single-Molecule Sensitivity in SurfaceEnhanced Raman Scattering. Phys. Rev. E - Stat. Physics, Plasmas, Fluids, Relat. Interdiscip. Top. 2000, 62 (3 B), $4318-$ 4324. https://doi.org/10.1103/PhysRevE.62.4318.

(16) Félidj, N.; Aubard, J.; Lévi, G.; Krenn, J. R.; Hohenau, A.; Schider, G.; Leitner, A.; Aussenegg, F. R. Optimized SurfaceEnhanced Raman Scattering on Gold Nanoparticle Arrays. Appl. Phys. Lett. 2003, 82 (18), 3095-3097. https://doi.org/10.1063/1.1571979.

(17) Le Ru, E. C.; Etchegoin, P. G. Single-Molecule Surface-Enhanced Raman Spectroscopy. Annu. Rev. Phys. Chem. 2012, 63 (1), 65-87. https://doi.org/10.1146/annurev-physchem-032511-143757.

(18) McFarland, A. D.; Young, M. A.; Dieringer, J. A.; Van Duyne, R. P. Wavelength-Scanned Surface-Enhanced Raman Excitation Spectroscopy. J. Phys. Chem. B 2005, 109 (22), 11279-11285. https://doi.org/10.1021/jp050508u.

(19) Yoshida, K. I.; Itoh, T.; Biju, V.; Ishikawa, M.; Ozaki, Y. Experimental Evaluation of the Twofold Electromagnetic Enhancement Theory of Surface-Enhanced Resonance Raman Scattering. Phys. Rev. B - Condens. Matter Mater. Phys. 2009, 79 (8), 085419. https://doi.org/10.1103/PhysRevB.79.085419.

(20) Khlebtsov, B. N.; Khanadeev, V. A.; Burov, A. M.; Le Ru, E. C.; Khlebtsov, N. G. Reexamination of Surface-Enhanced Raman Scattering from Gold Nanorods as a Function of Aspect Ratio and Shape. J. Phys. Chem. C 2020, 124 (19), 1064710658. https://doi.org/10.1021/acs.jpcc.0c00991.

(21) Nemati, A.; Wang, Q.; Hong, M.; Teng, J. Tunable and Reconfigurable Metasurfaces and Metadevices. Opto-Electronic Adv. 2018, 1 (5), 18000901-18000925. https://doi.org/10.29026/oea.2018.180009.

(22) Serien, D.; Sugioka, K. Fabrication of Three-Dimensional Proteinaceous Micro- and Nanostructures by Femtosecond Laser Cross-Linking. Opto-Electronic Adv. 2018, 1 (3), 18000801-18000818. https://doi.org/10.29026/oea.2018.180008.

(23) Suzuki, M. Practical Applications of Thin Films Nanostructured by Shadowing Growth. J. Nanophotonics 2013, 7, 073598. https://doi.org/10.1117/1.JNP.7.073598.

(24) Suzuki, M.; Wada, Y.; Maekita, W.; Nakajima, K.; Kimura, K.; Fukuoka, T.; Mori, Y. Physically Self-Assembled Ag Nanorod Arrays for Tunable Plasmonic Sensors. e-Journal Surf. Sci. Nanotechnol. 2005, 3, $280-283$. https://doi.org/10.1380/ejssnt.2005.280.

(25) Yan, D.; You-yi, S.; Kajii, H.; Maki, H.; Kin, Z.; Nawroth, T.; Johnson, R.; Krebs, L.; Suzuki, M.; Maekita, W.; et al. Direct Formation of Arrays of Prolate Ag Nanoparticles by Dynamic Oblique Deposition. Japanese J. Appl. Physics, Part 2 Lett. 2005, 44 (1-7), L193-L195. https://doi.org/10.1143/JJAP.44.L193.

(26) Rajput, A.; Kumar, S.; Singh, J. P. Vertically Standing Nanoporous Al-Ag Zig-Zag Silver Nanorod Arrays for Highly Active SERS Substrates. Analyst 2017, 142 (20), 3959-3966. https://doi.org/10.1039/c7an00851a.

(27) Suzuki, M.; Maekita, W.; Wada, Y.; Nakajima, K.; Kimura, K.; Fukuoka, T.; Mori, Y. Surface Enhanced Raman Scattering on Physically Self-Assembled Ag Nanorod Arrays. In Materials Research Society Symposium Proceedings; 2005 ; Vol. 900, pp 217-222. https://doi.org/10.1557/proc-0900-o08-03. 
Y.; et al. In-Line Aligned and Bottom-up Ag Nanorods for Surface-Enhanced Raman Spectroscopy. Appl. Phys. Lett. 2006, 88, 203121. https://doi.org/10.1063/1.2205149.

(29) Suzuki, M.; Maekita, W.; Wada, Y.; Nagai, K.; Nakajima, K.; Kimura, K.; Fukuoka, T.; Mori, Y. Ag Nanorod Arrays Tailored for Surface-Enhanced Raman Imaging in the near-Infrared region. Nanotechnology 2008, 19, 265304. https://doi.org/10.1088/0957-4484/19/26/265304.

(30) Kumar, S.; Goel, P.; Singh, D. P.; Singh, J. P. Highly Sensitive Superhydrophobic Ag Nanorods Array Substrates for Surface Enhanced Fluorescence Studies. Appl. Phys. Lett. 2014, 104, 023107. https://doi.org/10.1063/1.4861836.

(31) Kumar, S.; Goel, P.; Singh, D. P.; Singh, J. P. Fabrication of Superhydrophobic Silver Nanorods Array Substrate Using Glancing Angle Deposition. In AIP Conference Proceedings; 2014; Vol. 1591, p 872. https://doi.org/10.1063/1.4872786.

(32) Suzuki, M.; Akajima, K. N.; Imura, K. K.; Ukuoka, T. F.; Ori, Y. M. Au Nanorod Arrays Tailored for Surface-Enhanced Raman. Anal. Sci. 2007, 23 (July), 829-833.

(33) Kumar, S.; Doi, Y.; Namura, K.; Suzuki, M. Plasmonic Nanoslit Arrays Fabricated by Serial Bideposition: Optical and Surface-Enhanced Raman Scattering Study. ACS Appl. Bio Mater. 2020, 3, 3226-3235. https://doi.org/10.1021/acsabm.0c00215.

(34) Li, S.; Suzuki, M.; Nakajima, K.; Kimura, K.; Fukuoka, T.; Mori, Y. An Approach to Self-Cleaning SERS Sensors by Arraying Au Nanorods on TiO 2 Layer. Nanocoatings 2007, 6647, 66470J. https://doi.org/10.1117/12.731991.

(35) Suzuki, M.; Imai, Y.; Tokunaga, H.; Nakajima, K.; Kimura, K.; Fukuoka, T.; Mori, Y. Tailoring Coupling of Light to Local Plasmons by Using Ag Nanorods/Dielectric Layer/Mirror Sandwich Structures. In Proc. SPIE 7041, Nanostructured Thin Films; 2008; Vol. 7041. https://doi.org/10.1117/12.792750.

(36) Suzuki, M. Practical Applications of Thin Films Nanostructured by Shadowing Growth. J. Nanophotonics 2013, 7, 073598. https://doi.org/10.1117/1.JNP.7.073598.

(37) Ye, D.-X.; Karabacak, T.; Picu, R. C.; Wang, G.-C.; Lu, T.-M. Uniform Si Nanostructures Grown by Oblique Angle Deposition with Substrate Swing Rotation. Nanotechnology 2005, 16 (9), 1717-1723. https://doi.org/10.1088/0957$4484 / 16 / 9 / 052$.

(38) Bass, M.; DeCusatis, C.; Li, G.; Mahajan, V. N.; Enoch, J.; Stryland, E. V; of America, O. S. Handbook of Optics: Optical Properties of Materials, Nonlinear Optics, Quantum Optics. Handbook of Optics. McGraw-Hill 2009, p.

(39) Tikhonravov, A. V.; Baumeister, P. W.; Popov, K. V. Phase Properties of Multilayers. Appl. Opt. 1997, 36 (19), 4382. https://doi.org/10.1364/ao.36.004382.

(40) Birke, R. L.; Lombardi, J. R.; Gersten, J. I. Observation of a Continuum in Enhanced Raman Scattering from a MetalSolution Interface. Phys. Rev. Lett. 1979, 43 (1), 71-75. https://doi.org/10.1103/PhysRevLett.43.71.

(41) Ikeda, K.; Suzuki, S.; Uosaki, K. Enhancement of SERS Background through Charge Transfer Resonances on Single Crystal Gold Surfaces of Various Orientations. J. Am. Chem. Soc. 2013, 135 (46), 17387-17392. https://doi.org/10.1021/ja407459t.

(42) Mahajan, S.; Cole, R. M.; Speed, J. D.; Pelfrey, S. H.; Russell, A. E.; Bartlett, P. N.; Barnett, S. M.; Baumberg, J. J. Understanding the Surface-Enhanced Raman Spectroscopy “ Background.” J. Phys. Chem. C 2010, 114 (16), $7242-7250$. https://doi.org/10.1021/jp907197b.

(43) Farcau, C.; Astilean, S. Evidence of a Surface Plasmon-Mediated Mechanism in the Generation of the SERS Background. Chem. Commun. 2011, 47 (13), 3861-3863. https://doi.org/10.1039/c0cc05190j.

(44) Le Ru, E. C.; Etchegoin, P. G.; Ru, E. C. Le; Etchegoin, P. G.; Le Ru, E. C.; Etchegoin, P. G. Quantifying SERS Enhancements. MRS Bull. 2013, 38 (08), 631-640. https://doi.org/10.1557/mrs.2013.158.

Stiles, P. L.; Dieringer, J. A.; Shah, N. C.; Van Duyne, R. P. Surface-Enhanced Raman Spectroscopy. Annu. Rev. Anal. Chem. 2008, 1 (1), 601-626. https://doi.org/10.1146/annurev.anchem.1.031207.112814.

(46) Kumar, S.; Namura, K.; Suzuki, M. Proposal for a Gel-Based SERS Sensor. Proc. SPIE 2019, $10894,1089414$. https://doi.org/https://doi.org/10.1117/12.2506951. 
(47) Kumar, S.; Goel, P.; Singh, J. P. Flexible and Robust SERS Active Substrates for Conformal Rapid Detection of Pesticide Residues from Fruits. Sensors Actuators B Chem. 2017, 241, 577-583. https://doi.org/10.1016/J.SNB.2016.10.106. 


\section{Supporting Information to:}

\section{Experimental Evidence of Twofold Electromagnetic}

\section{Enhancement Mechanism of Surface-Enhanced}

\section{Raman Scattering}

Samir Kumar*, Kouta Tokunaga, Kyoko Namura, Takao Fukuoka, and Motofumi Suzuki**

Department of Micro Engineering, Graduate School of Engineering, Kyoto University, Katsura, Nishikyo, Kyoto 615-8540 Japan.

*Email: drsamirkumar2017@gmail.com,

**Email: m-snki@me.kyoto-u.ac.jp 


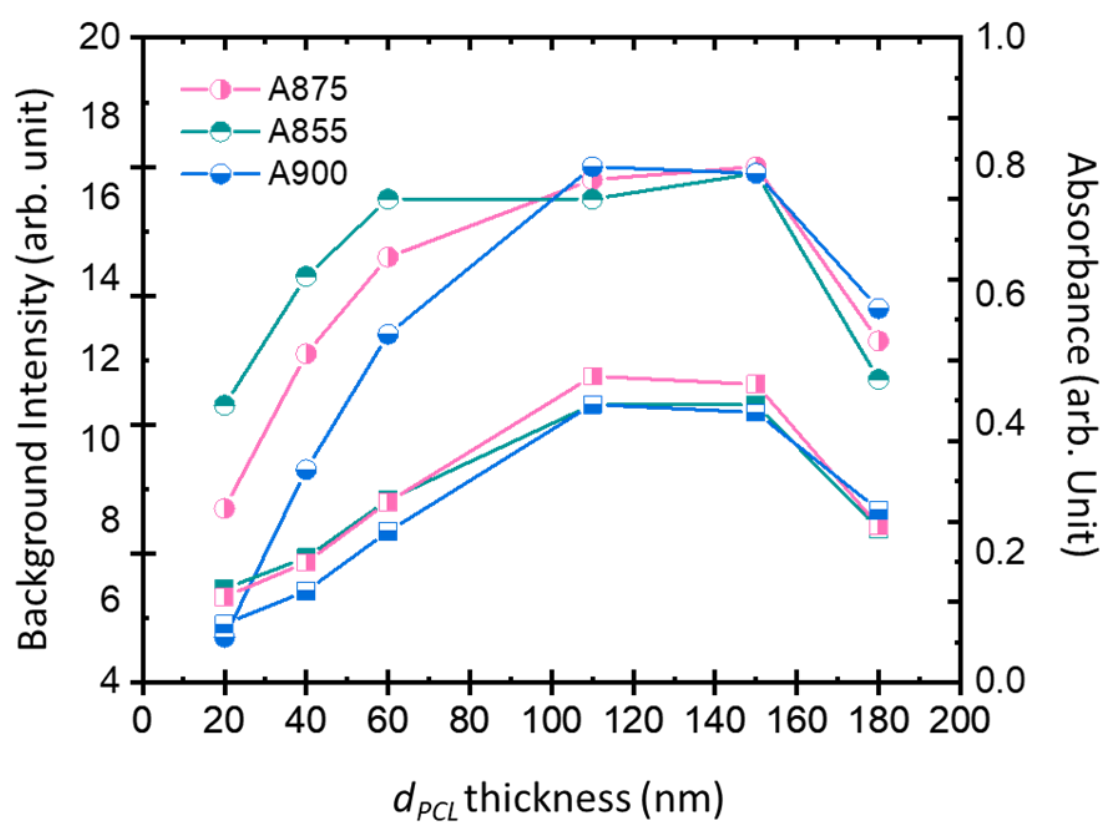

Figure S1. Absorbance (circular legend), background Intensity (square legend) vs. PCL thickness at the three Raman peaks corresponding to the wavelengths $855 \mathrm{~nm}$ (green), $875 \mathrm{~nm}$ (pink), and $900 \mathrm{~nm}$ (blue). There is a strong correlation between background enhancement and the absorbance spectra profile. Points to the role of surface plasmons in the generation of the SERS background 


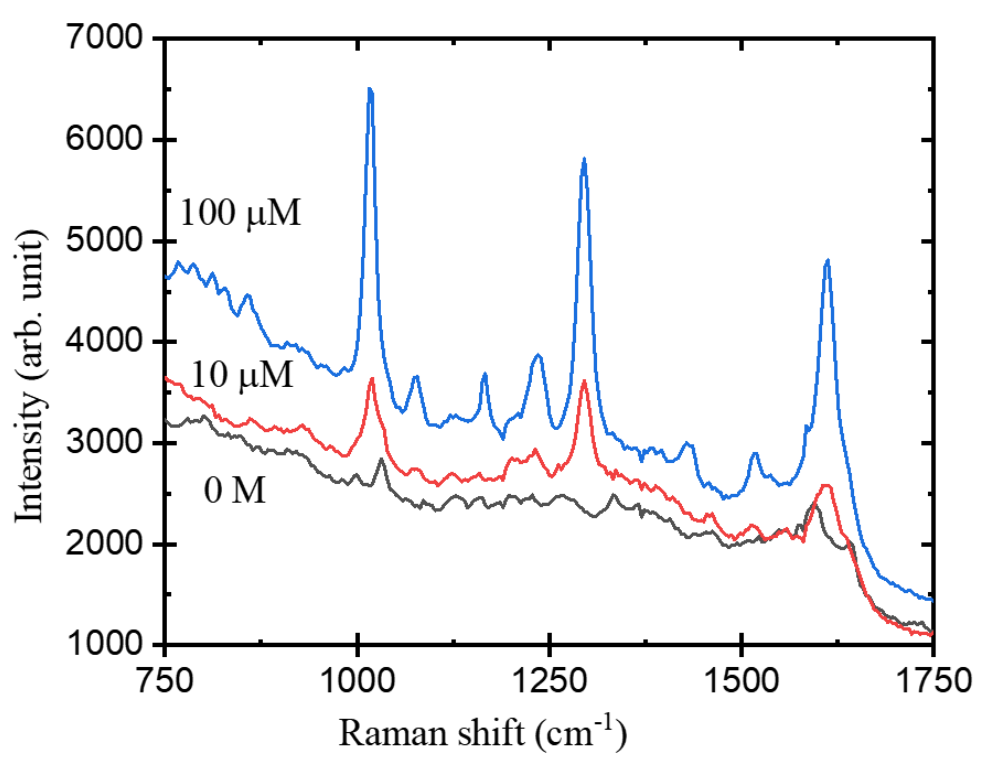

Figure S2. SERS spectra of BPY at various concentrations. The background is independent of the BPY concentration. This confirms that the absorption corresponds to the strength of the induced dipole, and one can thus identify a plasmonic mechanism involved in the generation of the SERS background. 

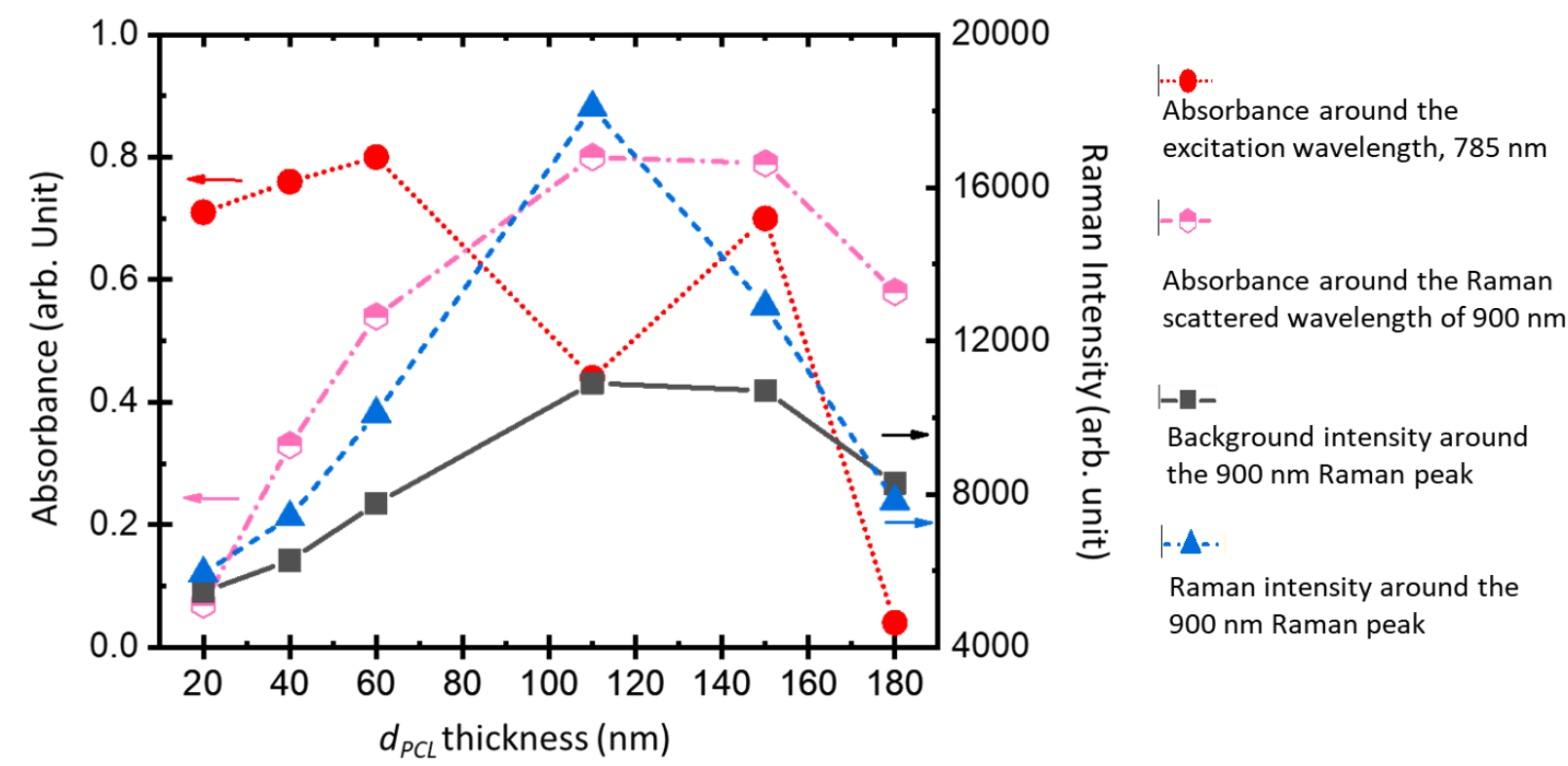

Figure S3. Absorbance, background intensity, and SERS peak intensity around $900 \mathrm{~nm}$ and excitation wavelength (785 $\mathrm{nm})$ as a function of PCL thickness. Raman peak intensity also follows the absorbance spectra profile. Absorbance around the excitation wavelength has an insignificant effect on the SERS and background spectra.

1

(1) Suzuki, M.; Imai, Y.; Tokunaga, H. Tailoring Coupling of Light to Local Plasmons by Using Ag Nanorods / Structured Dielectric / Mirror Sandwiches. J. Nanophotonics 2009, 3 (1), 1-12. https://doi.org/10.1117/1.3079804. 